\title{
Research on the Primary Features of the Internet of Things System and the Corresponding Data Communication Characteristics based on Sparse Coding and Joint Deep Neural Network
}

\author{
Jianping PAN $^{1, a}$, Wenzhun $\mathrm{HUANG}^{2, \mathrm{~b}^{*}}$ and Shanwen $\mathrm{ZHANG}^{2, \mathrm{c}}$ \\ ${ }^{1}$ Technology Department, Taiyuan Satellite Launch Center, Taiyuan 030027, \\ China \\ ${ }^{2}$ Department of Electronic Information Engineering, Xijing University, Xi'an \\ 710123, China

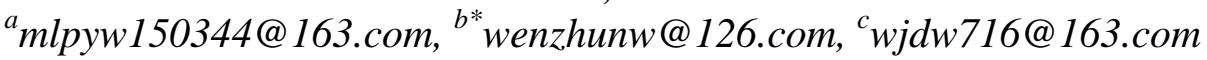 \\ *Corresponding author
}

\begin{abstract}
In this paper, we conduct research on issues related to the primary features of the Internet of things system and the corresponding data communication characteristics based on sparse coding and joint deep neural network. Internet of things is more than the underlying device difference communication method and it is the Internet of things needs to study in the field of hot issue. Using traditional algorithm for Internet communication equipment need particle filter was carried out on the acquisition of communication signal processing. Communication technology enables the Internet of things will perceive the information between different terminals for efficient transmission and exchange, exchange and sharing and the information resources is the key to the functions of things. To enhance the robustness and efficiency of the current IOT systems, we adopt the sparse coded dictionary learning theory to detect the size of the data and optimize the compressive sensing technique to modify the resolution. With the advances of the deep neural network, we analyze the topology of the system network structure and extract the pattern features and characteristics to make the signal transmission process more quickly and feasible. To enhance the objective function, we obtain the restricted optimization algorithm to help terminate the iteration for the higher efficiency. In the final part, we simulation our algorithm for times compared with other well-performed approaches. The result indicates that our method outperforms both in the accuracy layer an in the timeconsuming layer which will hold specific meaning.
\end{abstract}

Keywords: Internet of Things, Data Communication, Sparse Coding, Deep Neural Network, Signal Detection, Compressive Sensing, Feature Extraction, Mathematical Optimization

\section{Introduction}

With the rapid development of Internet technology, Internet of things in every industry plays an extremely important role. In the process of Internet technology, has changed the traditional way of communication. Traditional way of communication is often the case in large-scale data replication as the main operation process. And the Internet of things need for different kinds of information communication, therefore, need to select the most ideal way of huge amounts of data communication for more than the underlying data transmission equipment. So, Internet of things more than the underlying equipment difference method of communication. It has become the field of Internet of things need to study the hot issues which has received extensive concern of many scholars [1]. Internet 
of things more than the underlying device difference communication method, it is the Internet of things need to study in the field of hot issue. Using the traditional algorithm for Internet communication equipment, need particle filter was carried out on the acquisition of communication signal processing, control algorithm, and according to the process of the general communication signal transmission, so as to complete the basic Internet of things device communications. But assuming that the Internet of things the underlying many devices which will cause equipment compatibility degree of larger differences. Signals can't form the unified related requirements. Virtually increased the burden of communication and largely reduces the Internet of things more than the underlying equipment difference communication efficiency. Communication technology enables the use of the Internet of things perceived information the data between different terminals for efficient transmission and exchange, exchange and sharing, information resources is the key to the functions of things. Sensor can be regarded as the IOT of a peripheral network and perception stretch mesh. Sensor network is composed of multiple sensors, data processing unit and communication unit consisting of nodes through the organization way constitute a limited range of wireless local area network. The sensor network to offer Internet of things connected, and perception of information [2-9]. In general, the space structure and organization of the IOT could be summarized as the follows.

(1) Perceptual extension layer. Due to achieve in the Internet of things and things and communication of people and things, the perception layer is required. Perceptual extension layer is mainly to realize information collection to capture and object recognition. Perceptual extension layer must solve the problem of low power consumption, low cost and miniaturization and in the general core direction of higher sensitivity, more comprehensive awareness development.

(2) The network layer. Internet network layer consists of the main transmission of information. The network layer includes the access network and core network. Access to the Internet of things terminal to provide Internet access function, mobility management, etc., access network, including all kinds of cable access and the wireless access, etc. Core network will be largely based on the existing telecommunication network and the Internet. The network layer is the basic of Internet information hosted network. Existing various communication networks for the target customers and design, so the coexistence of diverse heterogeneous network formed the current situation. There are many kinds of equipment need to access the Internet, the Internet of things must be heterogeneous ubiquitous.

(3) The business and the application layer. The business and application layer information storage, data mining, the application of the decision-making, etc., ultimately to provide information and application of the business and the application layer of data storage and intelligent analysis, according to user to produce results. In the intelligent analysis layer, cloud computing is the core. With the development of the Internet application, the increase of the number of the terminal, cloud computing is needed to deal with the huge amounts of information, Internet of things to aid decision making and improve ability [10].

As a new networked computing system, the concept of Internet architecture and the architecture of networked computing system are given above. Therefore, in the design and implementation of Internet of things need to set up the Internet of things first before system structure, in order to make the final set up of the performance of the Internet of things system consistent with the expected demand. Establish IOT system structure of the main process of unified extracted from various and plenty application requirements of organization relationship between parts and the core components of the system. Strategies of network convergence can be understood as the basic network build public communication platform, a variety of heterogeneous networks realize the fusion of commonness and personality of synergy [11-15]. The so-called fusion is in common between the different systems integration, specific refers to all kinds of heterogeneous 
network and as a public communication platform of mobile communication network and the backbone transmission network or the fusion of next generation network, so as to constitute a ubiquitous net and coordination between the different systems is the integration of personality, refers to the net of the various access subnet through achieving coexistence, competition and cooperation between each other in order to satisfy the demands of business and application. In the figure one, we illustrate the core layers of the Internet of things system used for this paper.

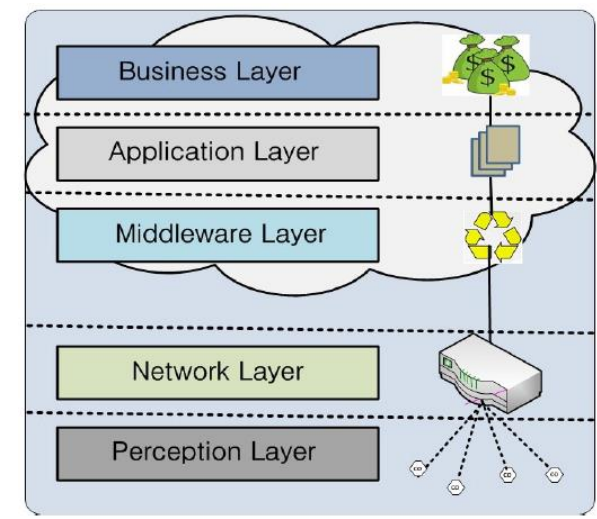

Figure 1. The Core Layers of the Internet of Things System Adopted

The energy distribution of sensor nodes in the monitoring area and computing resources are limited, and converge on the far side of the node has continuous energy supply and more powerful computing resources. The traditional data compression method coding and decoding is simple and at the same time, it means in the energy and the computing resource limited sensor nodes running complex coding algorithm, and in the focal point of the relatively rich resources to run the simple decoding algorithm. Traditional data compression methods are not symmetric and asymmetric sensor resources was not well. Using the network coding practical network data transmission system with buffer solution to the synchronization of the network coding theory hypothesis, the random linear network coding in a certain extent solved the theoretical assumptions in centralized network coding and network links have to demand, with the mentioned priority coding method such as section solves the theoretical assumptions in the network transmission speed to meet the requirements of minimum flow transfer node. In addition to these practical problems mentioned above, the practical network coding system also uses the more novel and effective method to theoretical results successfully applied to a variety of practical network transport problems. As long as the signal is compressible or in a transform domain is sparse which use base will not related observation matrix transformation and transformation project of high-dimensional signals to a low dimensional space, and then by solving an optimization problem can from these few related projection to reconstruct the original signal with high probability and reliability.

Sparse representation and dictionary learning is the hotspot in research of signal processing, different from the traditional processing method based on orthogonal transform, this method is based on the signal itself to study to get a group of atoms to represent the original signal, embodies the advantage when dealing with complex nonstationary signal. Constituted a series of atoms that are obtained through study the signal of the redundant dictionary, in the actual processing often want to signal the atomic number is less, the better, the original signal in the dictionary says is sparse, the sparse representation is also called sparse coding process. Modern signal processing theory, more and more applied to the study of spatial spectrum estimation, signal sparse decomposition as the new and innovative signal analysis thought which reflect its 
advantage has been applied in many fields. Signal sparse decomposition ideological and breakthrough of the current spatial spectrum analysis based on the limitations of orthogonal decomposition. Using the excellent characteristics of signal sparse representation, atomic parameters in the process of the decomposition and adaptive matching features can greatly improve the resolution of the spatial spectrum estimation and algorithm in low SNR environment estimation performance. Research in the application of spatial spectrum estimation, signal sparse decomposition has important practical significance. The figure two illustrates the coding concepts.

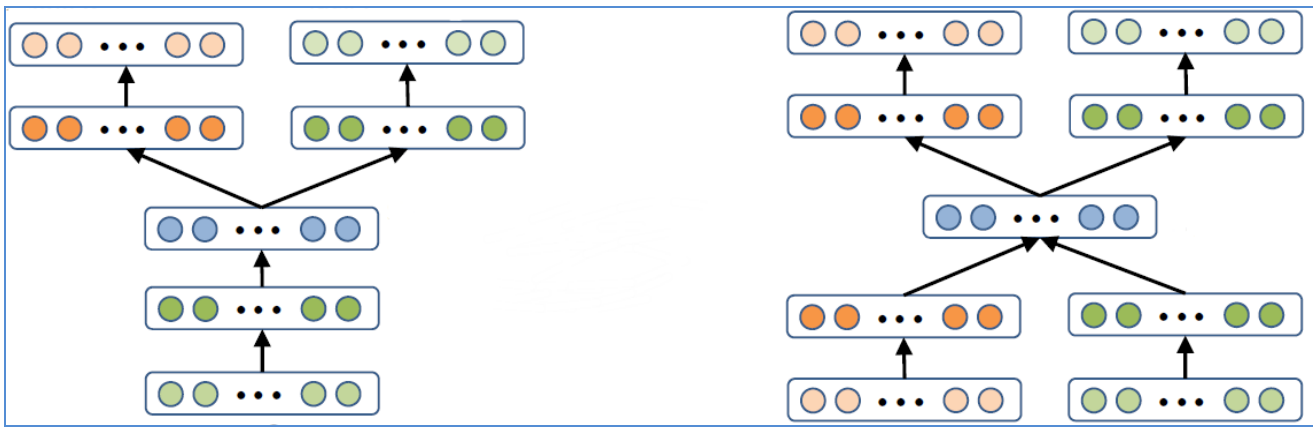

Figure 2. The Conceptual Demonstration of Sparse Coding Structure

In this paper, we analyze the issues related to the primary features of the Internet of things system and the corresponding data communication characteristics based on sparse coding and joint deep neural network. Deep learning research how to use multilayer structure automatic learning and potential distribution model data and it reduced the number of sample data and labeling requirements, through the training process of undirected automatic classification needs all levels of feature extracting which has the property of many different from existing machine learning techniques. The mobile communication terminal is in the core application of Internet of things. Mobile communication system of the mobile terminal as terminal equipment of information access which can with the mobile network information node, and to realize information between nodes and the network communication anytime and anywhere. Comparison of mobile communication terminal and IOT node information perception terminal function and way of working, the mobile communication can completely as the information node communication terminal parts used. This paper is organized as the follows. In the first section, we introduce the primary features of sparse coding, deep learning and the IOT. In the second section, we illustrate the basic theory of deep learning based neural network. In the third sections, we theoretically analyze the sparse coding algorithms and the dictionary learning. In the fourth part, we indicate the prior techniques based IOT communication technique. To bring the theoretical analysis into the experimental simulation, we conduct experiment in the section five. As the final conclusion, we summarize the research in last section.

\section{The Deep Neural Network}

\subsection{The General Introduction of the Deep Learning}

Deep learning is a new field of machine learning research, based on neural network model simulation analysis, the study of human brain, to imitate the brain mechanisms to identify the target and sensory information. Multi-layer perceptron network structure, for example, the entire network is an inseparable whole, between each neuron function, to study and deal with the data of the same or similar to each other, and basic function is to set up network mapping from input to output, unable or difficult to differentiate between 
input and output process of gradual transformation. Stereotypes neural network can be layered structure step by step, the basic function of the network divided into several independent functional modules. The network in the process of data processing is for a given concrete and the structure and parameters of network [16]. Deep learning has three main parts: the first, with unsupervised training system, which uses a large number of label sample extraction step by step, undirected automatic forming the characteristics. This process is similar to people through the sensory system receives the image such as eyes, ears, sound information, automatic forming different category information in the brain. Second, the alignment process, this process with some samples labeled with the characteristics of classification, and further adjusts the system parameters according to the results of the classification, to optimize the performance of the system on distinction between different categories of information. Similar to the one knows what is deer, what is a horse, or voice quality is good and what is bad. Third, test, use system has not seen the sample data inspection.

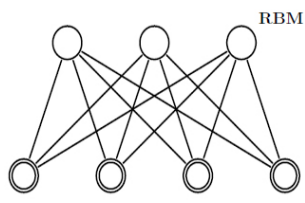

(a)

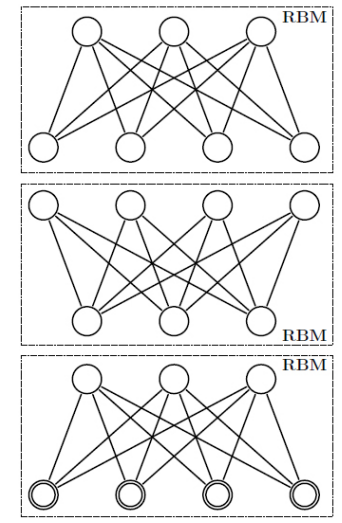

(b)

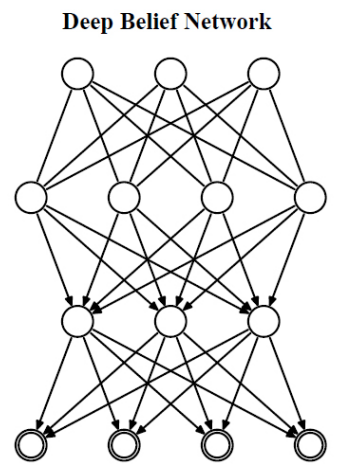

(c)

\section{Figure 3. The Structure and Inner Organization of the Deep Neural Network}

Algorithm is essentially a network error sum of squares of the objective function, according to the gradient method to minimize the objective function value algorithm. In addition to the input and output the final layer visible, depth study of other layers are hidden, which contains how much or how information is difficult to parse. It's like people saw an animal, imaging from the retina to the final judgement as a horse, in which abstract step by step through the nervous system, different sensory level of information is difficult to describe. To layered depth of training, however, must be clear each hidden layer the information, the provisions of corresponding relationship with the known quantity. Its theoretical basis, the physical concept is clear and made it possible to get all kinds of feed forward neural networks application. In the figure three, we demonstrate the basic structure and organization of the deep neural network.

\subsection{The Theoretical Description of the Deep Neural Network}

Cascading the automatic encoder based on deep learning can effectively extract the data low dimensional characteristics, is proposed in this paper the feature extraction is an important part of the classification model. About support vector machine method for solving classification problem, put forward is the central content structure risk minimum inductive principle, given the expansion of the machine learning ability of the upper bound estimate, to a method provided a very solid theoretical. But when a fixed structure, it will into pure planning problems, such as when take for hyperplane structure, the corresponding learning problem is generally reduced to solving the quadratic programming problem. Joint energy distribution function is shown below. 


$$
E(v, h)=-\sum_{i=1} \sum_{j=1}^{m} W_{i j} v_{i} h_{j}-\sum_{i=1}^{n} v_{i} b_{i}-\sum_{j=1}^{m} h_{j} c_{j}
$$

Learning matter's task is to calculate the values of these parameters to fit the given training data. The formula $2 \sim 3$ illustrate the conditional probability of hidden unit.

$$
\begin{gathered}
p\left(h_{j} \mid v\right)=\sigma\left(\sum_{i=1}^{n} w_{i j} v_{i}+c_{i}\right) \\
p\left(v_{j} \mid v\right)=\sigma\left(\sum_{i=1}^{n} w_{i j} h_{i}+b_{i}\right)
\end{gathered}
$$

Each layer of the training algorithm is the same: the bottom with known data as input to generate the data as the output. On its each layer, the following output paly as the input and generate data as the upper output. The nonlinear mapping ability and the characteristics of complex data said ability is weak, directly influenced the effect of data fusion complex deep model can approximation function, stronger generalization ability and data features said ability [17-19]. The formula four indicates the feature.

$$
\max _{x} \min _{k}\left\{\mid\left\langle x, c^{k}(i)\right\rangle\right\}, \quad i=1,2, \cdots, n \quad \text { s.t. } \quad C(i) x>0
$$

Planning study method on the basis of the algorithm based on search mechanism, the performance of the network as the optimization goal, constraint condition was used to optimize the network performance to improve the general ability to learn, than search mechanism based algorithm has superiority. In brief the method based on search, if do not change fundamentally, it is difficult to overcome its inherent shortcomings. Planning learning method and structural method obvious geometrical meaning of structural neural network is suitable for mass data processing. Planning algorithm because there are steps "optimization", therefore, in theory, the income of the network performance is better [20]. Coverage algorithm with the hyperplane of cutting sphere and spherical field of neuron structure neural network, classification ability, running speed can realize the division of multidisciplinary problem at the same time but it is suitable for the more problem of linear separable. The first layer classifier using rough set theory to a set into subsets, the rough set method can be well about attribute reduction of redundant information, extract the minimum core attribute set, effectively reduce the attribute dimension, and according to the non-numeric attributes in rough classification, would be in the first part of the subset of the user intent, regardless of the remaining subset, thereby reducing the number of samples of subsequent coverage algorithm. The second classification using coverage algorithm for the numeric data classification mode, play the advantages of covering algorithm, characteristics of numeric geometric model of the covers, accurately classify mode.

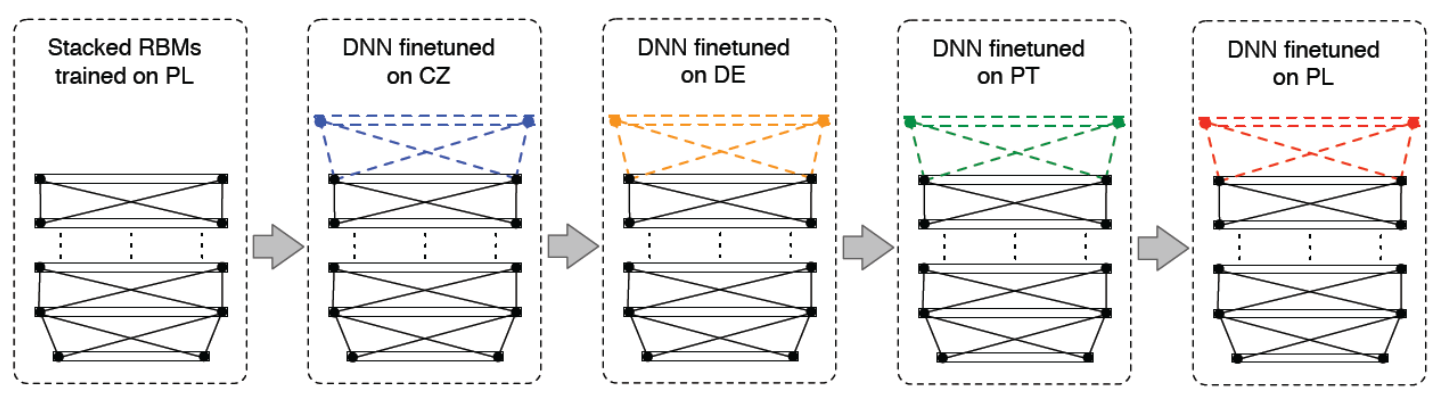

Figure 4.The Transmission Topology for Deep Neural Network 


\section{The Sparse Coding Algorithms and the Dictionary Learning}

\subsection{The Introduction to Compressive Sensing}

In the reality environment, a large number of real signals and the image itself is not sparse or compressible, but at a known base sparse or compressible. Traditional data coding method is after gathering the data, according to the sparse characteristic of signal on a sparse matrix, discard the small coefficient of the sparse matrix, realize the image compression. Traditional data encoding include "compression" after acquisition, first two steps, in the process of the front-end data acquisition collected a large number of redundant information, in the process of compression coding heavy calculation will be necessary to discard redundant information, caused the front-end data acquisition the node huge unnecessary burden [21]. Compressed sensing theory successfully integrate data acquisition namely to reduce the redundant information collected also reduce the computational complexity of the front-end data node. Therefore, the process of signal acquisition coding can be expressed as:

$$
Y=\Phi X=\Phi \Psi \theta=\prod \theta
$$

By introducing proper approximate calculation in news, constructs the general iterative receiver with logarithmic, computational complexity, its performance is superior to similar receiver is designed according to the heuristic method. For factor diagram and the algorithm to deal with continuous variables, the above studies are by Gaussian assumption of a single message passing algorithm, belongs to heuristic method. In the actual largescale wireless sensor networks, a large number of sensor measuring one or the general set of related signals at the same time, each set of sensor measurements in a certain set of base is sparse, and between various sensors and related. Signals will through linear projection to another and sparse matrix incoherent, on the base of each sensor separately encoding and transmission coefficient of a small number of results to collect nodes. Synergy between distributed compressed sensing does not require the acquisition node, according to get all of the measured value and we use the correlation of the global information or data to restore all the signals at the same time [22-25]. The formula 6 expresses the procedure.

$$
Y=\left(\begin{array}{c}
y_{1} \\
\vdots \\
y_{m}
\end{array}\right)=\left(\begin{array}{c}
y_{11}+\cdots+y_{1 n} \\
\vdots \\
y_{m 1}+\cdots+y_{m n}
\end{array}\right)=\Phi X=\left(\begin{array}{ccc}
\phi_{11} & \cdots & \phi_{1 m} \\
\vdots & \ddots & \vdots \\
\phi_{m 1} & \cdots & \phi_{m n}
\end{array}\right)\left(\begin{array}{c}
x_{1} \\
\vdots \\
x_{n}
\end{array}\right)
$$

Compressed sensing theory according to the sample dataset of "inner dimension" characteristics of the data acquisition, it is the mathematical basis of using small amounts of the adaptive linear measurement to relative can recover original signal measurement information and it will be the two general process data acquisition and coding consolidated into a linear projection process.

\subsection{The Sparse Coding Algorithms}

Sparse decomposition theory, the basic idea is: the functions with a library called atoms replace complete redundancy function system, the choice of atomic library conform to be as good as possible close to the structure of the signal, its composition which can be without any limitation, the elements in the library is called atoms. Found in the atomic library which has the best linear combination of muscle atoms to represent a signal which is known as a sparse approximation of signals. In the next following formulas, we define the optimal subset and approximation error.

$$
\begin{gathered}
\text { Optimal }=\operatorname{span}\left(g_{k}, \gamma \in I_{m}\right) \\
\sigma_{m}(f, D)=\inf _{f_{m}}\left\|f-f_{m}\right\|
\end{gathered}
$$


In the related redundant atom library to get the constructive results highly the nonlinear approximation, we must first focus on the particular atoms on the library. For a group of signal, choose different components have different joint sparse effect together [26]. If can know to the collected signals, each node, so can be best to identify common components make joint sparse representation of sparse degree is minimal. Based on this idea, combined with the application of sensor, adopts clustering hierarchical routing method, the transmission of data acquisition of cluster-heads node first to the cluster head nodes, cluster head nodes according to the data of each node to determine the best common component, and then to the projection of common components and characteristics respectively, the transmission of projection values to the gathering node and then in gathering the node joint recovery out signal vectors of each node. The demonstration of the distribution is shown in the figure 5 .

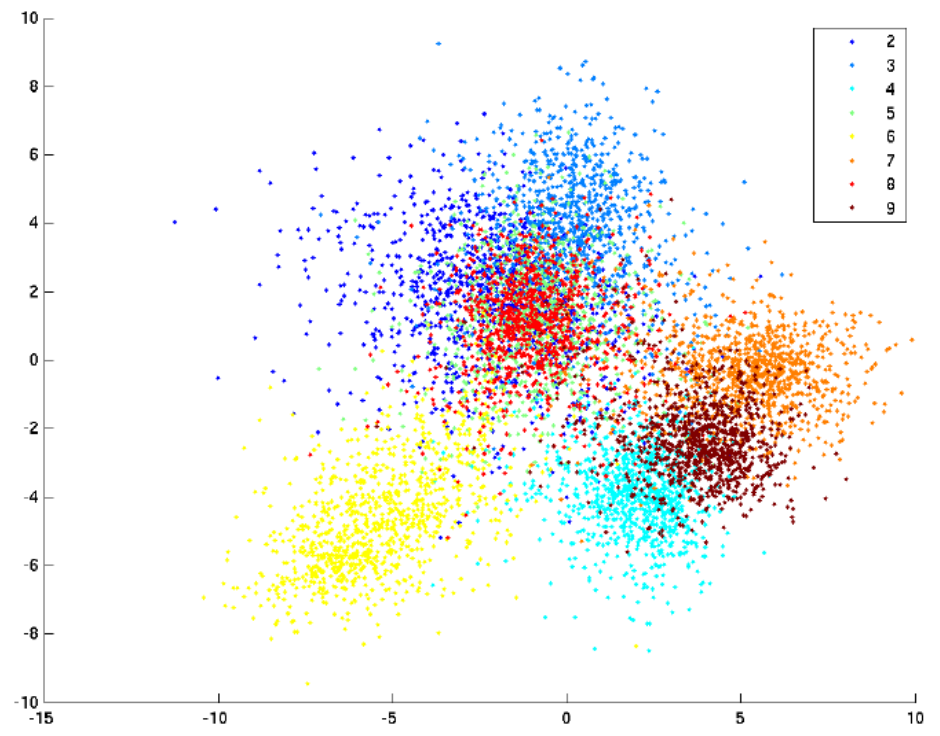

\section{Figure 5.The Demonstration of the Signal Sparsity for the Communication}

Through signal sample autocorrelation matrix decomposition and separately by the maximum and minimum eigenvalue two subspace with the signal subspace and noise subspaces. Construct two subspace of vector of the pseudo-spectral function on the sinusoidal frequency has a sharp peak, therefore can estimate the frequency of the sine wave. Based on the analysis of the general feature subspace algorithm is generally divided into signal subspace frequency estimation algorithm, and noise subspace frequency estimation algorithm. Signal subspace algorithm is to ignore the noise influence and only to keep the characteristics of the signal subspace vector information with noise space algorithm is based on the nature of the signal subspace and noise subspace orthogonal. The solution of proximal problem defined as follows.

$$
\min _{\alpha \in R^{p}} f(\hat{\alpha})+(\alpha-\hat{\alpha})^{\mathrm{T}}+\lambda \Omega(\alpha)+\frac{L}{2}\|(\alpha-\hat{\alpha})\|_{2}^{2}
$$

Redundancy backup mechanism is safe storage system to improve system reliability and fault tolerant ability is an important method of full backup, delete code and network coding are redundant backup mechanism of some important technology. We could therefore simplify the formula into the expression 10.

$$
\min _{\alpha \in R^{p}} \frac{1}{2}\left\|\alpha-\left(\hat{\alpha}-\frac{1}{L} \nabla f(\hat{\alpha})\right)\right\|_{2}^{2}+\lambda / 2 \times \Omega(\alpha)
$$

In this model, the node sampling the signal consists of common components and characteristics of addition. Contact is directly related to determine the common component of sparse representation of sparse effect and so best to identify common 
component is the key to the compressed sampling using the model. In the figure 6 , we show the general flowchart of the sparse coding algorithm.

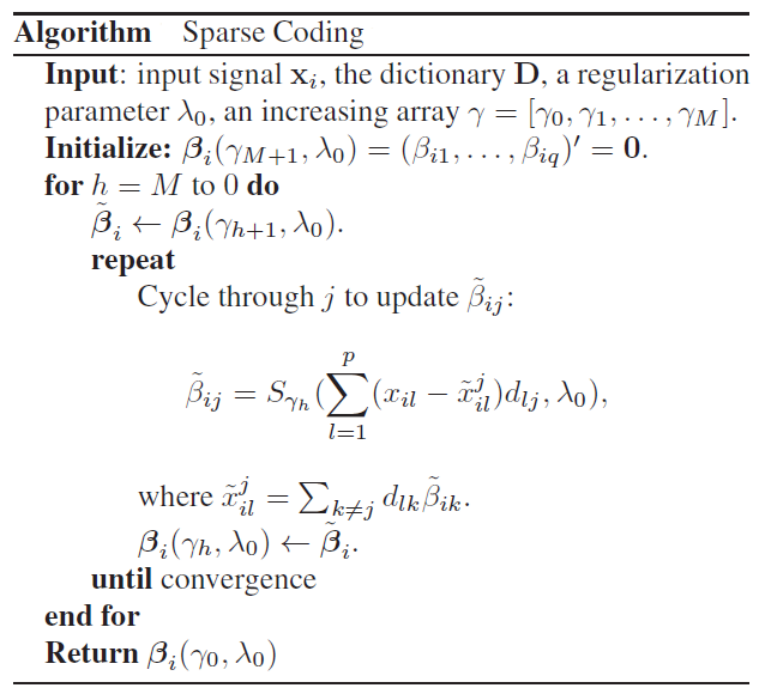

\section{Figure 6.The Flowchart of the Sparse Coding Algorithm}

\subsection{The Dictionary Learning Procedure with OMP}

Against LSP parameters for the training, first of all, finding the mean to mean the training after the LSP parameters to the dictionary learning, and training parameters of LSP parameter dictionary of sparse coding matrix. Use the OMP algorithm to go after the average test LSP parameters such as sparse decomposition in dictionary LSP parameters for testing parameters of the sparse matrix coding and then refactor out after the mean test LSP parameters. LT codes process is fairly simple. Generate the process of encoding symbols are as follows: first, from the degree distribution of prior decision randomly selected from a coding symbol, then the uniform random choose $d$ a different input symbols as symbols of adjacent nodes, and coded character set for this $\mathrm{d}$ an adjacent node or the value of the vision. Source generated according to the above process and in the transmission channel coding symbol, until the decoder to receive enough encoded symbols and decoding the source data recovery. In the following flowchart, we show the algorithm for the online dictionary learning.

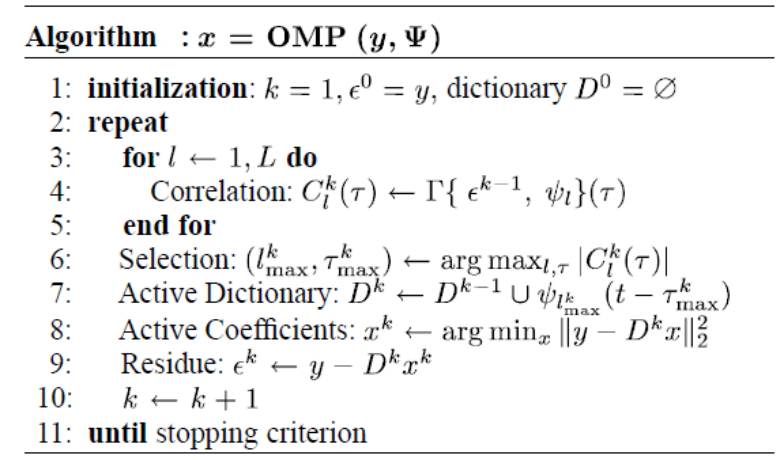

Figure 7.The Flowchart of the Dictionary Learning Algorithm

\section{Prior Knowledge based IOT Communication Technique}

The Internet of things system consists of hardware and software two architectural system. Core hosted network hardware subsystem consists of sensor network, and information service system of three parts. Among them, the sensor network including 
sensor network node and endings. The sensor node is mainly used for data acquisition and equipment control. Peripheral also known as access network, including the gathering node and access gateway, it is mainly used for peripheral sensor node of the network control and data gathering. The core carrying network is the basis of the Internet communication network, mainly used in the general access network and the communication between the information service systems. Internet of things is to make the object and the network real time together, through various means such as object recognition, positioning and analysis, get the useful information application in some fields. IOT scenarios, production in the life everywhere, the application of Internet of things technology is usually involved in various fields, multidisciplinary a kind of intelligent application of technology [27-29]. When in the mobile communication network construction planning first, plays an important role in the basic network construction, network planning is the foundation of network construction.

Usually network planning time is very long, need to determine the planning area and the core dimension, through field investigation. Distribution and the economic development of landform, building a comprehensive consideration, combined with the local user's lifestyle and living standards aspects of data for analysis. In the present physical distribution and the distribution of current network base station business network base station, on the basis of the simulation software, to predict and meet the needs of covering planning and capacity planning, determine the construction scale and site configuration. Network planning is to use the lowest cost of investment, construction meet the coverage during the planning period and the optimal network capacity development. Assume that the Internet of things used in the wireless sensor network using popular clustering structure, the base station is completely believable and the network attacker can configure some in the area of the legitimate nodes and the performance of the legitimate nodes as malicious nodes, or will make the malicious nodes after legal node capture. For any fusion process of neighboring nodes to collect information on the physical must have redundancy based on the statistics and information theory, by examining its sensory data report to measure the degree of each node can be trusted, by node credibility, each fusion results associated with a credibility evaluation, achieve the result of the fusion measurement uncertainty. In the next section, we will test and verify the mentioned technique and application on IOT.

\section{Numerical Experiment and the Simulation}

\subsection{Experimental Environment Initiation}

In this part, we analyze the initialized environment for later experiment. Research on the Internet of things is more than the underlying device difference of efficient communication method. The Internet has a variety of underlying device. Equipment directly there are big differences in compatibility, the different signals in the transmission speed, distance, the different requirements of power consumption, lead to the signal transmission cannot form the unified requirements, the traditional communication technology using multi-protocol method according to the different equipment set different protocols, virtually increased communication burden with the low efficiency. Therefore, in this experiment, we initialize the device in the mode of low which will be less energyconsuming. At bottom of the Internet of things devices communication signals, the need to block the acquisition of communication signal, it is divided into several sub band. Establish communication signal fusion rules for these sub bands. In order to verify the energy and weighted algorithm is proposed in this paper on the Internet of things the underlying equipment effectiveness of communication, the need for an experiment. In the next following formula 11, we demonstrate the measurement standard and policy for the experiment. 


$$
Q_{\text {Measurement }}=R_{\text {Sparse }}\left[\sqrt{\frac{\Theta_{\text {Sparse }}+\Theta_{\text {Dictionary }} / \sigma_{\text {Mean }}}{(K / Q P) \cos ^{2} \eta_{k}+\left(Q_{0} / 2 Q v_{f}\right)}}\right]
$$

\subsection{Simulation Result and Corresponding Analysis}

In this sub-section, we conduct simulation on the proposed approach. We first analyze the sparse coded dictionary learned features for the IOT system. In the figure 8 we visually demonstrate the result, we could see from the figure that the learned features varies from the space and the time scale which reflect the possibility for the IOT communication signals to be sparse. By Laplace energy and weighted algorithm, can true reflect the characteristics of the Internet communication signal intensity in the bottom to large extent can represent characteristics of communication signals and truth. Based on the clustering of the hierarchical routing protocol, data is the cluster head nodes, at the top of cluster heads to identify the common component with high possibility. Then the application of cluster nodes in the compressive sensing for the joint coding, finally complete joint decoding in converge on the far side of the node.

To verify the effectiveness and robustness of the proposed methodology, in figure 9 , we conduct individual experiment for 200 times with the comparison of the other state-ofthe-art algorithms. Compared the success rate of joint decoding and decoding refactoring alone, for every observation 2000 times repeated trials, the relative value of mean square error as the reconstruction error, if the reconstruction error is less than 0.02 , is seen as refactoring success, success to refactor the success rate for the ratio of the number and the total number of test. We could learn from the simulation result that the proposed method enhance the traditional methods in large extent.

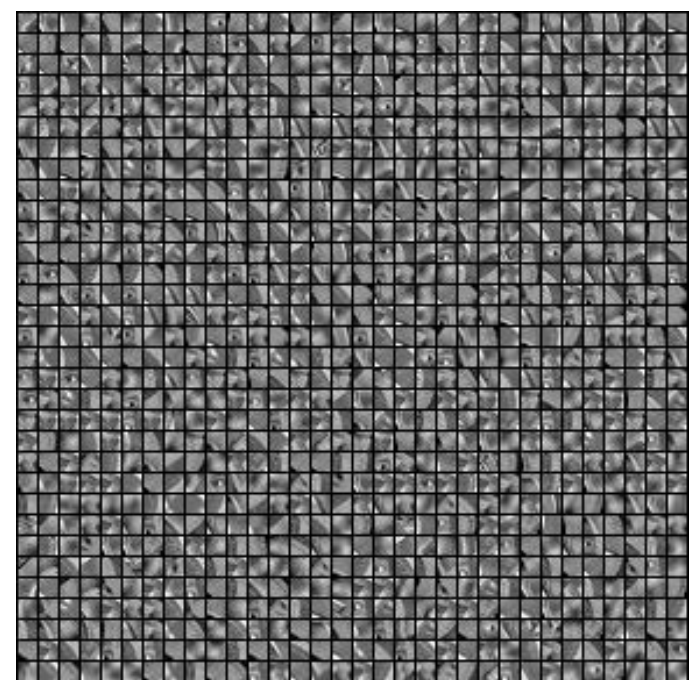

Figure 8.The Sparse Coded Dictionary Learned Features for the IOT System 

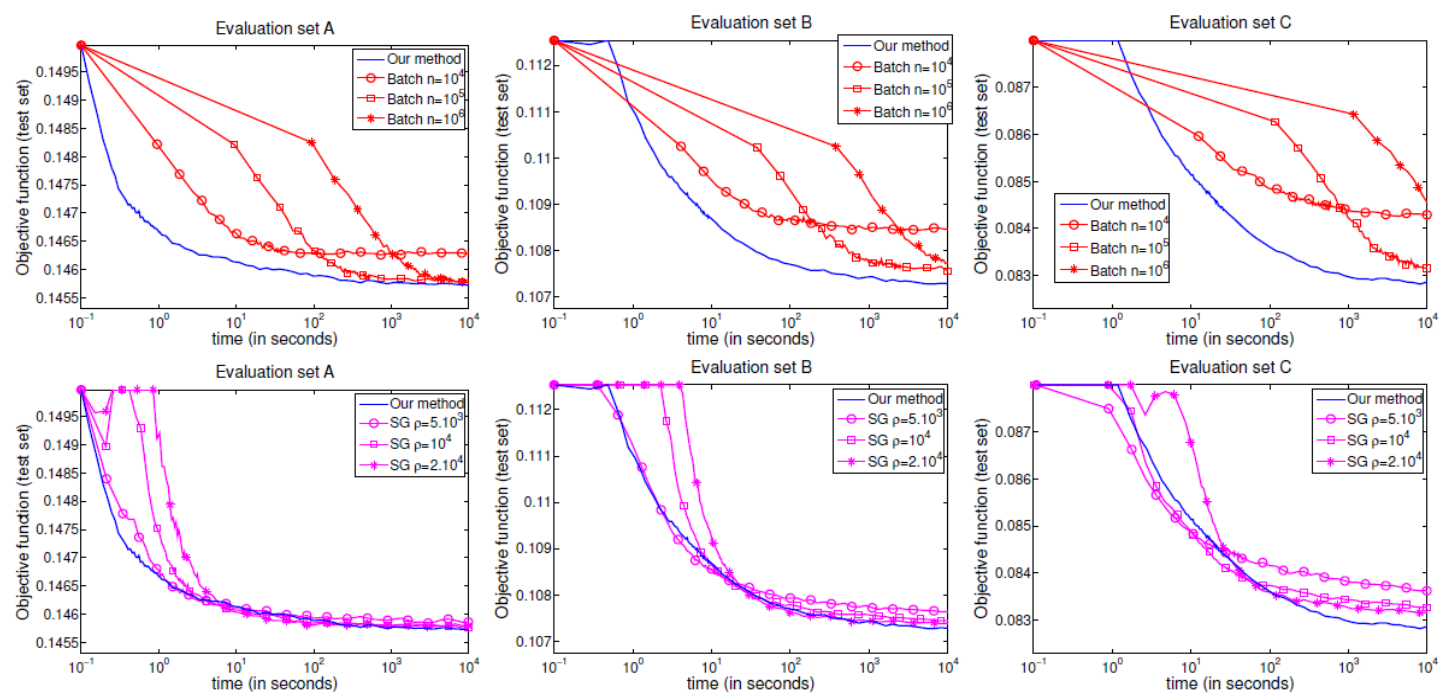

Figure 9.The Experimental Result for the Proposed Algorithm

\section{Conclusion and Summary}

In this paper, we analyze the issues related to the primary features of the Internet of things system and the corresponding data communication characteristics based on sparse coding and joint deep neural network. We need to point out that there is no unified analysis of these architecture, nor do layered inductive method to these, more not match the architecture and implementation method, and makes people now or in the development of IOT system is difficult to choose what kind of system structure and implementation methods for reference. During transition to the next generation network and the network is the inevitable trend of fusion with no clear boundaries of professional network now. This article from the perspective of spatial correlation of sensor nodes and build a differentia value signal sparse model, and on the premise of guarantee accurate reconstruction, discusses the feasibility of independent encoding, joint decoding, the corresponding distributed compressed sensing algorithm is given. The algorithm can be in node without communication to differential signal encoding, save the node energy consumption and balance the node energy balance, energy efficient way to meet the application of the Internet of things. The simulation result indicates the effectiveness of the proposed method. In the future, we plan to combine the convex optimization algorithm into the dictionary updating steps to modify the current approach to gain better performance.

\section{Acknowledgements}

This work is financially supported by the Project of National Natural Science Foundation of China (Program No. 61473237) and the scientific research program of Shaanxi Provincial Education Department (Program No. 14JK2156).

\section{References}

[1] D. Miorandi, S. Sicari, F. Pellegrini and I. Chlamtac, "Internet of things: Vision, applications and research challenges", Ad Hoc Networks 10, no. 7, (2012), pp. 1497-1516.

[2] F. Riggins and S. Wamba, "Research Directions on the Adoption, Usage, and Impact of the Internet of Things through the Use of Big Data Analytics", In System Sciences (HICSS), 2015 48th Hawaii International Conference on, (2015), pp. 1531-1540.

[3] K. Hwang, J. Dongarra and G. Fox, "Distributed and cloud computing: from parallel processing to the internet of things. Morgan Kaufmann, (2013). 
[4] A. Pal, A. Mukherjee and P. Balamuralidhar, "Model-Driven Development for Internet of Things: Towards Easing the Concerns of Application Developers", In Internet of Things. User-Centric IoT, (2015), pp. 339-346.

[5] M. Kovatsch, S. Mayer and B. Ostermaier, "Moving application logic from the firmware to the cloud: Towards the thin server architecture for the internet of things", In Innovative Mobile and Internet Services in Ubiquitous Computing (IMIS), 2012 Sixth International Conference on, (2012), pp. 751-756.

[6] L. Atzori, A. Iera and G. Morabito, "Siot: Giving a social structure to the internet of things", Communications Letters, IEEE 15, no. 11, (2011), pp. 1193-1195.

[7] J. Y. Duan, and F. Guo, "Research of Wireless Functions of Human Body Detection System Based on The Internet of Things", In 2015 International Conference on Advances in Mechanical Engineering and Industrial Informatics. Atlantis Press, (2015).

[8] D. Uckelmann, M. Harrison and F. Michahelles, "An architectural approach towards the future internet of things", In Architecting the internet of things, (2011), pp. 1-24.

[9] P. Vlacheas, R. Giaffreda, V. Stavroulaki, D. Kelaidonis, V. Foteinos, G. Poulios, P. Demestichas, A. Somov, A. Biswas and K. Moessner, "Enabling smart cities through a cognitive management framework for the internet of things", Communications Magazine, IEEE, vol. 51, no. 6, (2013), pp. 102-111.

[10] A. Jara, M. Zamora and A. Skarmeta, "Glowbal IP: An adaptive and transparent IPv6 integration in the Internet of Things", Mobile Information Systems, vol. 8, no. 3, (2012), pp. 177-197.

[11] M. Swan, T. Kido and Minna Ruckenstein., "BRAINY-Multi-modal Brain Training App for Google Glass: Cognitive Enhancement, Wearable Computing, and the Internet-of-Things extend Personal Data Analytics", In Workshop on Personal Data Analytics in the Internet of Things, 40th International Conference on Very Large Databases, Hangzhou, China, (2014).

[12] R. Roman, C. Alcaraz, J. Lopez and N. Sklavos, "Key management systems for sensor networks in the context of the Internet of Things", Computers \& Electrical Engineering, vol. 37, no. 2, (2011), pp. 147159.

[13] A. Sivieri, L. Mottola and G. Cugola, "ELIoT: Building Internet of Things Software Combining Localized and Internet-scale Interactions", Major revision submitted for publication on Computer Networks, (2013).

[14] H. Wang and J. Wang, "An effective image representation method using kernel classification", In Tools with Artificial Intelligence (ICTAI), 2014 IEEE 26th International Conference on, pp. 853-858. IEEE, (2014).

[15] M. Ramezani, P. Abolmaesumi, K. Marble, H. MacDonald and I. Johnsrude, "Joint sparse representation of brain activity patterns related to perceptual and cognitive components of a speech comprehension task", In Pattern Recognition in NeuroImaging (PRNI), 2012 International Workshop on, (2012), pp. 2932.

[16] X. Mei and H. Ling, "Robust visual tracking and vehicle classification via sparse representation", Pattern Analysis and Machine Intelligence, IEEE Transactions, vol. 33, no. 11, (2011), pp. 2259-2272.

[17] X. Jia, H. Lu and H. H. Yang, "Visual tracking via adaptive structural local sparse appearance model", In Computer vision and pattern recognition (CVPR), 2012 IEEE Conference on, (2012), pp. 1822-1829.

[18] D. Donoho, Y. Tsaig, I. Drori and J. Starck, "Sparse solution of underdetermined systems of linear equations by stage wise orthogonal matching pursuit", Information Theory, IEEE Transactions on, vol. 58, no. 2, (2012), pp. 1094-1121.

[19] D. Xu, Y. Huang, Z. Zeng and X. Xu, "Human gait recognition using patch distribution feature and locality-constrained group sparse representation”, Image Processing, IEEE Transactions on, vol. 21, no. 1, (2012), pp. 316-326.

[20] M. Yang, L. Zhang, X. Feng and D. Zhang, "Sparse representation based fisher discrimination dictionary learning for image classification", International Journal of Computer Vision, vol. 109, no. 3 (2014), pp. 209-232.

[21] Z. Wang, J. Xie, G. Zhou, Z. Ji and T. Quan, "Real-valued sparse representation for single snapshot direction-of-arrival estimation in shipborne high-frequency surface wave radar", IET Radar, Sonar \&amp; Navigation, (2015).

[22] H. Zhang, Y. Zhang and T Huang, "Simultaneous discriminative projection and dictionary learning for sparse representation based classification", Pattern Recognition, vol. 46, no. 1, (2013), pp. 346-354.

[23] J. Yin and T. Chen, "Direction-of-arrival estimation using a sparse representation of array covariance vectors", Signal Processing, IEEE Transactions on, vol. 59, no. 9, (2011), pp. 4489-4493.

[24] E. Ortiz, A. Wright and M. Shah, "Face recognition in movie trailers via mean sequence sparse representation-based classification', In Computer Vision and Pattern Recognition (CVPR), 2013 IEEE Conference on, (2013), pp. 3531-3538.

[25] Z. Huang, Y. Liu, C. Li, M. Yang and L. Chen, "A robust face and ear based multimodal biometric system using sparse representation”, Pattern Recognition, vol. 46, no. 8, (2013), pp. 2156-2168.

[26] I. Theodorakopoulos, D. Kastaniotis, G. Economou and S. Fotopoulos, "Pose-based human action recognition via sparse representation in dissimilarity space", Journal of Visual Communication and Image Representation, vol. 25, no. 1, (2014), pp. 12-23. 
[27] H. Zhang, J. Yang, Y. Zhang, N. Nasrabadi and T. Huang, “Close the loop: Joint blind image restoration and recognition with sparse representation prior'. In Computer Vision (ICCV), 2011 IEEE International Conference on, (2011), pp. 770-777.

[28] C. Jung, L. Jiao, H. Qi and T. Sun, "Image deblocking via sparse representation”, Signal Processing: Image Communication, vol. 27, no. 6, (2012), pp. 663-677.

[29] Y. Xu, Z. Fan, M. Qiu, D. Zhang and J. Y. Yang, "A sparse representation method of bimodal biometrics and palm print recognition experiments", Neurocomputing, vol. 103, (2013), pp. 164-171. 